\title{
Time Series Forecasting Using Novel Feature Extraction Algorithm and Multilayer Neural Network
}

\author{
Raheleh Rezazadeh \\ Master student of software Engineering, Ferdows \\ Islamic Islamic Azad University, \\ Ferdows, Iran
}

\author{
Hooman Kashanian \\ Department of Computer science and software \\ Engineering \\ Islamic Azad University, \\ Ferdows, Iran
}

\begin{abstract}
Time series forecasting is important because it can often provide the foundation for decision making in a large variety of fields. A tree-ensemble method, referred to as time series forest (TSF), is proposed for time series classification. The approach is based on the concept of data series envelopes and essential attributes generated by a multilayer neural network... These claims are further investigated by applying statistical tests. With the results presented in this article and results from related investigations that are considered as well, we want to support practitioners or scholars in answering the following question: Which measure should be looked at first if accuracy is the most important criterion, if an application is time-critical, or if a compromise is needed? In this paper demonstrated feature extraction by novel method can improvement in time series data forecasting process.
\end{abstract}

Keyword: time series data, neural network, forecasting 


\section{INTRODUCTION}

Classical statistics and data analysis primarily address items that can be described by a classic variable that takes either a real value (for a quantitative variable) or a category (for a nominal variable). However, observations and estimations in the real world are usually not sufficiently complete to represent classic data exactly. In the stock market, for instance, stock prices have their daily (or weekly, or monthly) bounds and vary in each period (be it a day, week, or month). Representing the variations with snapshot points (e.g., the closing price) only reflects a particular number at a particular time; it does not properly reflect its variability during the period. This problem can be eased if the highest and lowest prices per period are considered, giving rise to interval-valued data. Interval-valued data is a particular case of symbolic data in the field of symbolic data analysis (SDA) [14]. SDA states that symbolic variables (lists, intervals, frequency distributions, etc.) are better suited than single-valued variables for describing complex real-life situations [2]. It should be noted that interval-valued data in the field of SDA does not come from noise assumptions but rather from the expression of variation or aggregation of huge databases into a reduced number of groups [22]. When considering a chronological sequence of interval-valued data, interval time series (ITS) arises quite naturally. Modeling and forecasting of ITS has the advantage of taking into account the variability and/or uncertainty, and it reduces Therefore, tools for ITS forecasting are very much in demand. According to the existing literature, the main methodologies available for ITS forecasting fall roughly into two categories in relation to the method in which interval data is handled, i.e., splitting single-valued methods or interval-valued methods. For the first category, the lower and upper bounds of interval data are treated as two independent single-valued parts, such as the autoregressive integrated moving average (ARIMA) employed in [3]. For the second category, the lower and upper bounds of interval data are treated using interval arithmetic as interval-valued data, as in the interval Holt's exponential smoothing method (HoltI) [22], the vector auto regression/vector error correction model [11,16], multilayer perceptron (MLP) [22], and interval MLP (IMLP) [21]. Interested readers are referred to [1] for a recent survey of the presented methodologies and techniques employed for ITS forecasting. In this study, we propose to take the form of complex numbers to represent interval data, i.e., by denoting the lower and upper bounds of the interval as real and imaginary parts of a complex number, respectively, thus allowing us to use the complex-valued neural network (CVNN) for ITS prediction. The CVNN is a type of neural network in which weights, threshold values, and input and output signals are all complex numbers. The activation function as well as its derivatives have to be "well behaved" everywhere in the complex plane [20]. CVNNs exhibit very desirable characteristics in their learning, self-organizing, and processing dynamics. This, together with the widespread use of analytic signals, gives them a significant advantage in practical applications in diverse fields of engineering, where signals are routinely analyzed and processed in time/space, frequency, and phase domains. A significant number of studies have demonstrated that CVNNs have better capabilities than real-valued neural networks for function approximation [17,20] and classification tasks [16,17]. Due to the localization ability and simple architecture of radial basis function (RBF) neural networks in the real domain, the complex-valued RBF neural network is gaining interest among researchers. Notable earlier work includes that of Chen et al. [9], who investigated a complex-valued RBF neural network with complex-valued weights and a real-valued activation function using several learning algorithms. Other related studies can be found in Jianping et al. [20] and Deng et al. [13]. Complex-valued RBF neural networks typically use a Gaussian activation function that maps complex-valued inputs to a real-valued hyper-dimensional feature space at the hidden layer. However, as the mapping is done at the hidden layer, the input is not efficiently transmitted to the output [15], which results in inaccurate phase approximation [12]. To overcome the limitations, researchers have started to develop fully complex-valued regression methods (or classifiers) for solving real valued function approximation (or classification) problems. Recently, a fully complex-valued RBF neural network (FCRBFNN) using a hyperbolic secant function as the activation function was derived by Savitha et al. [7]. Their experimental study clearly showed that the FCRBFNN can outperform other complex-valued RBF networks from the literature for function approximation [4]. In view of the FCRBFNN's advantages in processing complex-valued signals, it will be interesting to investigate the possibility of forecasting the lower and upper bounds of ITS in the form of complex intervals using the FCRBFNN. Another issue considered in this study is the evolution of structure (or topology) and parameters (e.g., scaling factors and weights) of the FCRBFNN. In general, the learning steps of a neural network are as follows. First, a network structure is determined with a predefined number of inputs, hidden nodes, and outputs. Second, an algorithm is chosen to realize the learning process. In [30], for instance, the number of hidden nodes was first determined by the K-means clustering algorithm, and then a fully complex-valued gradient descent learning algorithm was used to tune the FCRBFNN. Since the gradient descent algorithm, may get stuck in local optima and is highly dependent on the starting points, research efforts have been made on using evolutionary computation methods to design and evolve neural networks [10,12,11,9,3]. Following this line of research, in this study, we use multilayer neural network (MNN) [21]. 


\section{Time Series Data Models and Forecasting}

Time series forecasting, or time series prediction, takes an existing series of data $x_{t-n}, \ldots, x_{t-2}, x_{t-1}, x_{t}$ and forecasts the $x_{t+1}, x_{t+2}, \ldots$ data values. The goal is to observe or model the existing data series to enable future unknown data values to be forecasted accurately. Examples of data series include financial data series (stocks, indices, rates, etc.), physically observed data series (sunspots, weather, etc.), and mathematical data series (Fibonacci sequence, integrals of differential equations, etc.). The phrase "time series" generically refers to any data series, whether or not the data are dependent on a certain time increment. Throughout the literature, many techniques have been implemented to perform time series forecasting. This paper will focus on two techniques: neural networks and k-nearest-neighbor. This paper will attempt to fill a gap in the abundant neural network time series forecasting literature, where testing arbitrary neural networks on arbitrarily complex data series is common, but not very enlightening. This paper thoroughly analyzes the responses of specific neural network configurations to artificial data series, where each data series has a specific characteristic. A better understanding of what causes the basic neural network to become an inadequate forecasting technique will be gained. In addition, the influence of data preprocessing will be noted. The forecasting performance of k-nearest-neighbor, which is a much simpler forecasting technique, will be compared to the neural networks' performance. Finally, both techniques will be used to forecast a real data series. Time series Models and forecasting methods have been studied by various people and detailed analysis can be found in [Error! Reference source not found., Error! Reference source not found.,Error! Reference source not found.]. Time Series Models can be divided into two kinds. Univariate Models where the observations are those of single variable recorded sequentially over equal spaced time intervals. The other kind is the Multivariate, where the observations are of multiple variables. A common assumption in many time series techniques is that the data are stationary. A stationary process has the property that the mean, variance and autocorrelation structure do not change over time. Stationarity can be defined in precise mathematical terms, but for our purpose we mean a flat looking series, without trend, constant variance over time, a constant autocorrelation structure over time and no periodic fluctuations. There are a number of approaches to modeling time series. We outline a few of the most common approaches below. Trend, Seasonal, Residual Decompositions: One approach is to decompose the time series into a trend, seasonal, and residual component. Triple exponential smoothing is an example of this approach. Another example, called seasonal loess, is based on locally weighted least squares. Frequency Based Methods: Another approach, commonly used in scientific and engineering applications, is to analyze the series in the frequency domain. An example of this approach in modeling a sinusoidal type data set is shown in the beam deflection case study. The spectral plot is the primary tool for the frequency analysis of time series.

Autoregressive (AR) Models: A common approach for modeling univariate time series is the autoregressive (AR) model equation (1):

$X_{l}=\delta+\phi_{1} X_{t-1}+\phi_{2} X_{t-2}+\ldots+\phi_{p} X_{t-p}+A_{t}$

where $\mathrm{Xt}$ is the time series, at is white noise, and

$\delta=\left(1-\sum_{i=1}^{p} \phi_{i}\right) \mu$

with $\boldsymbol{L}$ denoting the process mean.

An autoregressive model is simply a linear regression of the current value of the series against one or more prior values of the series. The value of $p$ is called the order of the AR model. AR models can be analyzed with one of various methods; including standard linear least squares techniques. They also have a straightforward interpretation. Moving Average (MA): Models another common approach for modeling univariate time series models is the moving average (MA) model:

$X_{i}=\mu+A_{i}-\theta_{1} A_{i-1}-\theta_{2} A_{i-2}-\ldots-\theta_{q} A_{i-q}$

where $\mathrm{Xt}$ is the time series, $\boldsymbol{L}$ is the mean of the series, $\mathbf{A}_{\mathrm{t}-\mathrm{i}}$ are white noise, and $1, \ldots, \mathrm{q}$ are the parameters of the model. The value of $\mathrm{q}$ is called the order of the MA model.

That is, a moving average model is conceptually a linear regression of the current value of the series against the white noise or random shocks of one or more prior values of the series. The random shocks at each point are assumed to 
come from the same distribution, typically a normal distribution, with location at zero and constant scale. The distinction in this model is that these random shocks are propagated to future values of the time series. Fitting the MA estimates is more complicated than with AR models because the error terms are not observable. This means that iterative non-linear fitting procedures need to be used in place of linear least squares. MA models also have a less obvious interpretation than AR models. Note, however, that the error terms after the model is fit should be independent and follow the standard assumptions for a univariate process.

Box-Jenkins Approach: The Box-Jenkins ARMA model is a combination of the AR and MA models:

$\mathrm{X}_{t}=\delta+\phi_{1} X_{t-1}+\phi_{2} X_{t-2}+\ldots+\phi_{p} X_{t-p}+$

$A_{i}-\theta_{1} A_{i-1}-\theta_{2} A_{i-2}-\ldots-\theta_{q} A_{i-q}$

where the terms in the equation have the same meaning as given for the AR and MA model [Error! Reference source

not found.].

The Box-Jenkins model assumes that the time series is stationary. Box and Jenkins recommend differencing nonstationary series one or more times to achieve stationarity. Doing so produces an ARIMA model, with the "I" standing for "Integrated". Some formulations transform the series by subtracting the mean of the series from each data point. This yields a series with a mean of zero. Whether you need to do this or not is dependent on the software you use to estimate the model. Box-Jenkins models can be extended to include seasonal autoregressive and seasonal moving average terms. Although this complicates the notation and mathematics of the model, the underlying concepts for seasonal autoregressive and seasonal moving average terms are similar to the non-seasonal autoregressive and moving average terms. The most general Box-Jenkins model includes difference operators, autoregressive terms, moving average terms, seasonal difference operators, seasonal autoregressive terms, and seasonal moving average terms. As with modeling in general, however, only necessary terms should be included in the model.

\subsection{Steps in the Time Series Forecasting Process:}

The goal of a time series forecast is to identify factors that can be predicted. This is a systematic approach involving the following steps and show in Figure (1).

Step 1: Hypothesize a form for the time series model.

Identify which of the time series components should be included in the model.

Perform the following operations.

Collect historical data.

Graph the data vs. time.

Hypothesize a form for the time series model.

Verify this hypothesis statistically.

Step 2: Select a forecasting technique. A forecasting technique must be chosen to predict future values of the time series.

The values of input parameters must be determined before the technique can be applied.

Step 3: Prepare a forecast.

The appropriate data values must be substituted into the selected forecasting model.

The forecast may be affected by Number of past observations used. 
Initial forecast value used.

The following flowchart highlights the systematic development of the modeling and forecasting phases:

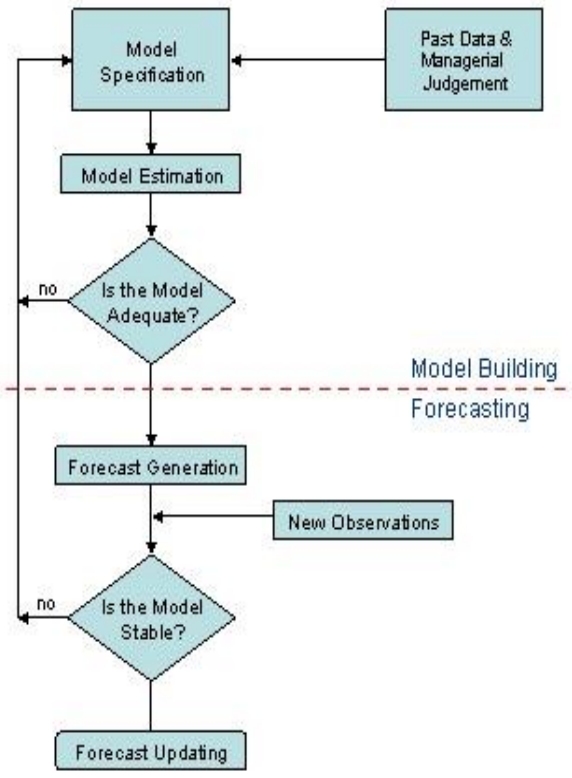

Figure (1): time series forecasting architecture

Stationary Forecasting Models: In a stationary model the mean value of the time series is assumed to be constant.

\subsection{Feature Extraction Algorithm}

The proposed feature extraction scheme processes the magnetic, angular rate, and accelerometer signals provided by the MARG sensors in order to excerpt 1. the orientation of the person w.r.t. the earth frame, and 2. the acceleration in the person frame, $\mathrm{P}$ a. In contrast to other feature extraction schemes [4, 7], we consider that angular rate measurements provided by gyroscopes are not valuable signals any longer for the classification algorithms, since their information is incorporated to the orientation of the person. Therefore, the main goal consists in computing $\mathrm{P} \mathrm{E}^{\wedge} \mathrm{q}$, i.e., the orientation of the earth frame $(\mathrm{E})$ relative to the person frame $(\mathrm{P})$. The proposed algorithm makes use of quaternion property 2 ., decomposing the estimation of $\mathrm{P} \mathrm{E}^{\wedge} \mathrm{q}$ as a concatenation of the estimation of the orientation of Ez w.r.t. to $\mathrm{P} z, \mathrm{P} \mathrm{E}^{\wedge} \mathrm{qz}$, followed by the estimation of the orientation of the plane Exy w.r.t. the plane $\mathrm{P} x y, \mathrm{P} \mathrm{E}^{\wedge} \mathrm{qxy}$, i.e.,

$$
\begin{aligned}
& \stackrel{\mathrm{P} \wedge}{\mathrm{E}} \mathrm{q}={ }_{\mathrm{E}}^{\mathrm{P}} \mathrm{q}_{\mathrm{xy}} \otimes{ }_{\mathrm{E}}^{\mathrm{P} \wedge} \mathrm{q}_{\mathrm{z}} \\
& \text { where }{ }_{\mathrm{E}}^{\mathrm{P}} \mathrm{q}_{\mathrm{z}} \text { is also decomposed as } \\
& \stackrel{\mathrm{P} \wedge}{\mathrm{E}} \mathrm{q}_{\mathrm{z}}={ }_{\mathrm{E}}^{\mathrm{S}} \mathrm{q} \otimes{ }_{\mathrm{S}}^{\mathrm{P}} \mathrm{q}_{\mathrm{z}}
\end{aligned}
$$

Algorithm 1 summarizes the process to compute $\mathrm{P} \mathrm{E}^{\wedge} \mathrm{q}[\mathrm{n}]$, the orientation of the earth frame w.r.t. the person frame. The calculation is performed for the $\mathrm{N}$ available samples of magnetic field, angular rate, and acceleration measurements acquired by the MARG sensor. Note that _, the key parameter of the sensor orientation algorithm [6] must be selected at the beginning, and it plays a key role in the performance of the classification algorithm. 


\begin{tabular}{|c|c|c|c|c|c|c|}
\hline \multicolumn{7}{|c|}{ Algorithm 1 Pseudocode of person orientation algorithm } \\
\hline \multicolumn{7}{|l|}{ for $\mathrm{n}=1: \mathrm{N}$ do } \\
\hline \multicolumn{7}{|c|}{ Compute $\underset{E}{S} \wedge \mathrm{q}[\mathrm{n}]$ with the algorithm of [6] and $\beta$} \\
\hline \multicolumn{7}{|c|}{ Detect whether the person is walking } \\
\hline \multicolumn{7}{|c|}{ Update ${ }_{\mathrm{S}}^{\mathrm{P}} \wedge \mathrm{q}_{\mathrm{z}}[\mathrm{n}]$} \\
\hline \multicolumn{7}{|c|}{ Update ${ }_{\mathrm{E}}^{\mathrm{P}} \mathrm{q}_{\mathrm{XY}}[\mathrm{n}]$} \\
\hline \multicolumn{7}{|c|}{${ }_{\mathrm{S}}^{\mathrm{P}} \mathrm{q}_{\mathrm{z}}[\mathrm{n}]={ }_{\mathrm{S}}^{\mathrm{P} \wedge} \mathrm{q}_{\mathrm{z}}[\mathrm{n}-1]$} \\
\hline \multicolumn{7}{|c|}{ end if } \\
\hline \multicolumn{7}{|c|}{${ }_{\mathrm{E}}^{\mathrm{P}} \mathrm{q}[\mathrm{n}]={ }_{\mathrm{E}}^{\mathrm{P} \wedge} \mathrm{q}_{\mathrm{XY}}[\mathrm{n}] \otimes_{\mathrm{E}}^{\mathrm{S} \wedge} \mathrm{q}[\mathrm{n}] \otimes_{\mathrm{S}}^{\mathrm{P} \wedge} \mathrm{q}_{\mathrm{Z}}[\mathrm{n}]$} \\
\hline \multicolumn{7}{|l|}{ end for } \\
\hline $\begin{array}{l}2.3 \\
2.4 \\
2.5\end{array}$ & & & & & & \\
\hline \multicolumn{7}{|c|}{ Table 1Beginning parameters for heuristically trained neural networks. } \\
\hline \multicolumn{7}{|c|}{$\begin{array}{l}\text { Heuristic Algorithm Training } \\
\text { Update Frequency }=\mathbf{5 0} \text {, Change Frequency }=10, \text { Decrement }=0.05\end{array}$} \\
\hline Architecture & $\begin{array}{l}\text { Learning } \\
\text { Rate }\end{array}$ & $\begin{array}{l}\text { Epochs } \\
\text { Limit }\end{array}$ & $\begin{array}{l}\text { Error } \\
\text { Limit }\end{array}$ & $\begin{array}{l}\text { Data Series } \\
O=\text { original } \\
\mathbf{L}=\text { less noisy } \\
\mathbf{M}=\text { more noisy } \\
\mathbf{A}=\text { ascending }\end{array}$ & $\begin{array}{lr}\text { Training } & \text { Set } \\
\text { Data Point } \\
\text { Range (\# of } \\
\text { Examples) }\end{array}$ & $\begin{array}{l}\text { Validation Set } \\
\text { Data Point Range } \\
\text { (\# of Examples) }\end{array}$ \\
\hline $35: 20: 1$ & 0.3 & 500,000 & $1 \times 10-10$ & $\mathrm{O}, \mathrm{L}, \mathrm{M}, \mathrm{A}$ & $0-143(109)$ & $144-215(37)$ \\
\hline $35: 10: 1$ & 0.3 & 500,000 & $1 \times 10-10$ & $\mathrm{O}, \mathrm{L}, \mathrm{M}, \mathrm{A}$ & $0-143(109)$ & $144-215(37)$ \\
\hline $35: 2: 1$ & 0.3 & 500,000 & $1 \times 10-10$ & $\mathrm{O}, \mathrm{L}, \mathrm{M}, \mathrm{A}$ & $0-143(109)$ & $144-215(37)$ \\
\hline $25: 20: 1$ & 0.3 & 250,000 & $1 \times 10-10$ & $\mathrm{O}$ & $0-143(119)$ & $144-215(47)$ \\
\hline $25: 10: 1$ & 0.3 & 250,000 & $1 \times 10-10$ & $\mathrm{O}$ & $0-143(119)$ & $144-215(47)$ \\
\hline
\end{tabular}


Table 2 beginning parameter

\begin{tabular}{|c|c|c|c|c|c|c|}
\hline \multicolumn{7}{|c|}{ Simple Method Training } \\
\hline Architecture & $\begin{array}{l}\text { Learning } \\
\text { Rate }\end{array}$ & $\begin{array}{l}\text { Epochs } \\
\text { Limit }\end{array}$ & $\begin{array}{l}\text { Error } \\
\text { Limit }\end{array}$ & $\begin{array}{l}\text { Data Series } \\
O=\text { original } \\
L=\text { less noisy } \\
M=\text { more noisy } \\
A=\text { ascending } \\
S=\text { sunspots }\end{array}$ & $\begin{array}{l}\text { Training } \\
\text { Data Set } \\
\text { Range Point } \\
\text { Examples) }\end{array}$ & $\begin{array}{l}\text { Validation Set } \\
\text { Data Point Range } \\
\text { (\# of Examples) }\end{array}$ \\
\hline $35: 20: 1$ & 0.1 & 100,000 & $1 \times 10-10$ & $\mathrm{O}, \mathrm{L}, \mathrm{M}, \mathrm{A}$ & $0-143(109)$ & $144-215(37)$ \\
\hline $35: 10: 1$ & 0.1 & 100,000 & $1 \times 10-10$ & $\mathrm{O}, \mathrm{L}, \mathrm{M}, \mathrm{A}$ & $0-143(109)$ & $144-215(37)$ \\
\hline $35: 2: 1$ & 0.1 & 100,000 & $1 \times 10-10$ & $\mathrm{O}, \mathrm{L}, \mathrm{M}, \mathrm{A}$ & $0-143(109)$ & $144-215(37)$ \\
\hline $30: 30: 1$ & 0.05 & 100,000 & $1 \times 10-10$ & $\mathrm{~S}$ & $0-165(136)$ & - \\
\hline
\end{tabular}

Often, two trained networks with identical beginning parameters can produce drastically different forecasts. This is because each network is initialized with random weights and biases prior to training, and during training, networks converge to different minima on the training error curve. Therefore, three candidates of each network configuration were trained. This allows another neural network forecasting technique to be used: forecasting by committee. In forecasting by committee, the forecasts from the three networks are averaged together to produce a new forecast. This may either smooth out noisy forecasts or introduce error from a poorly performing network. The coefficient of determination will be calculated for the three networks' forecasts and for the committee forecast to determine the best candidate.

\section{Multi Neural Network Parameters and Procedure Architectures}

To test how feed-forward neural networks respond to various data series, a network architecture that could accurately learn and model the original series is needed. An understanding of the feed-forward neural network is necessary to specify the number of network inputs. The trained network acts as a function: given a set of inputs it calculates an output. The network does not have any concept of temporal position and cannot distinguish between identical sets of inputs with different actual outputs. For example, referencing the original data series, if there are ten network inputs, among the training examples (assuming the training set covers an entire period) there are several instances where two examples have equivalent input vectors but different output vectors. One such instance is shown in Figure . This may "confuse" the network during training, and fed that input vector during forecasting, the network's output may be somewhere in-between the output vectors' values or simply "way off"!

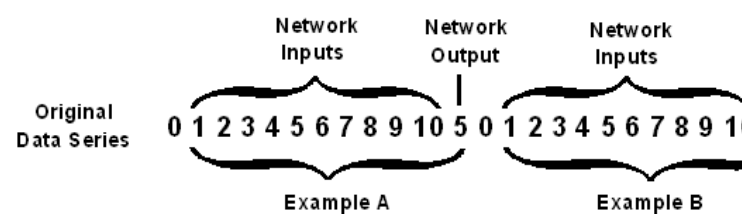

Figure 2 One instance in the original data series

where two examples have equivalent input vectors but

different output vectors.

Inspection of the original data series reveals that a network with at least twenty-four inputs is required to make unambiguous examples. (Given twenty-three inputs, the largest ambiguous example input vectors would be from zero-based data point 10 to 32 and from 34 to 56.) Curiously, considerably more network inputs are required to make good forecasts, as will be seen in Section Error! Reference source not found.

Next, through trial-and-error the number of hidden layers was found to be one and the number of units in that layer was found to be ten for the artificial data series. The number of output layer units is necessarily one. This network, called 35:10:1 for shorthand, showed excellent forecasting performance on the original data series, and was selected to be the reference for comparison and evaluation. To highlight any effects of having a too-small or too-large network for the task, two other networks, 35:2:1 and 35:20:1, respectively, are also included in the evaluation. Also, addressing the curiosity raised in the previous 
paragraph, two more networks, 25:10:1 and 25:20:1, are included.

It is much more difficult to choose the appropriate number of network inputs for the sunspots data series. By inspecting the data series and through trial-anderror, thirty network inputs were selected. Also through trial-and-error, one hidden layer with thirty units was selected. Although this network seems excessively large, it proved to be the best neural network forecaster for the sunspots data series. Other networks that were tried include 10:10:1, 20:10:1, 30:10:1, 10:20:1, 20:20:1, and 30:20:1.

\section{Training}

By observing neural network training characteristics, a heuristic algorithm was developed and implemented in FORECASTER. The parameters for the heuristic are set within the Training Parameters dialog (see Section Error! Reference source not found.). The heuristic requires the user to set the learning rate and epochs limit to higher-than-normal values (e.g., 0.3 and 500,000 , respectively) and the error limit to a lowerthan-normal value (e.g., $1 \times 10^{-10}$ ). The heuristic also uses three additional user-set parameters: the number of training epochs before an application window (view) update (update frequency), the number of updates before a learning rate change (change frequency), and a learning rate change decrement (decrement). Finally, the heuristic requires the data series to be partitioned into a training set and validation set. Given these users set parameters, the heuristic algorithm is: for each view-update during training

if the validation error is higher than the lowest value seen

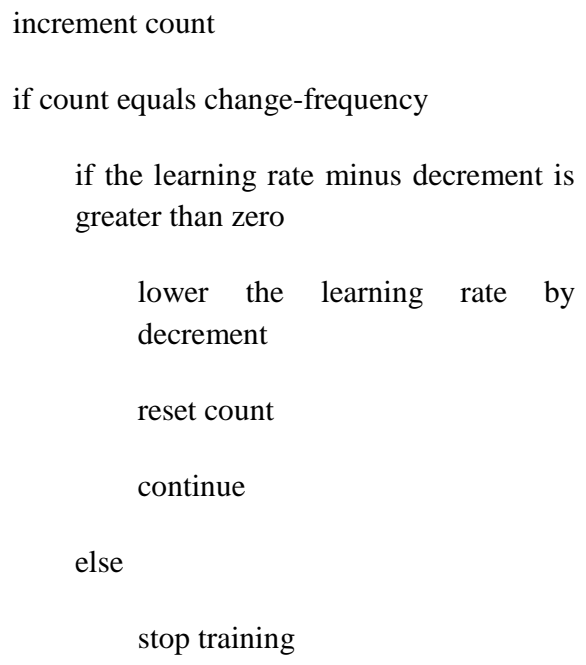

The purpose of the heuristic is to start with an aggressive learning rate, which will quickly find a coarse solution, and then to gradually decrease the learning rate to find a finer solution. Of course, this could be done manually by observing the validation error and using the Change Training Parameters dialog to alter the learning rate. But an automated solution is preferred, especially for an empirical evaluation.

In the evaluation, the heuristic algorithm is compared to the "simple" method of training where training continues until either the number of epochs grows to the epochs limit or the total squared error drops to the error limit. Networks trained with the heuristic algorithm are termed "heuristically trained"; networks trained with the simple method are termed "simply trained".

Finally, the data series in Section Error! Reference source not found. are partitioned so that the training set is the first two periods and the validation set is the third period. Note that "period" is used loosely for less noisy, noisier, and ascending, since they are not strictly periodic.

\section{Simply Trained Neural Networks with Thirty-Five Inputs}

Figure graphically shows the one-period forecasting accuracy for the best candidates for simply trained 35:2:1, 35:10:1, and 35:20:1 networks. Figure (a), (b), and (c) show forecasts from networks trained on 
the original, less noisy, and more noisy data series, respectively, and the forecasts are compared to a period of the original data series. Figure (d) shows forecasts from networks trained on the ascending data series, and the forecasts are compared to a fourth "period" of the ascending series. In Figure (c) the 35:2:1 network is not included.

Figure graphically compares metrics for the best candidates for simply trained 35:2:1, 35:10:1, and 35:20:1 networks. Figure (a) and (b) compare the total squared error and unscaled error, respectively, and (c) compares the coefficient of determination. The vertical axis in (a) and (b) is logarithmic. The number of epochs and training time are not included in Figure because all networks were trained to 100,000 epochs.

Finally, Table lists the raw data for the charts in Figure and other parameters used in training. Refer to Error! Reference source not found. for more parameters.

In this case, it seems the heuristic was inappropriate. Notice that the heuristic allowed the 35:2:1 network to train for 28,850 epochs more than the simple method. Also, the total squared error and unscaled error for the heuristically trained network were lower, but so was the coefficient of determination it was much lower. Again, the forecasts for the 35:10:1 and 35:20:1 networks are near perfect, and are indistinguishable from the original data series.

In Figure (b) the 35:2:1 network forecast is worse than in Error! Reference source not found. (b), whereas the 35:10:1 and 35:20:1 forecasts are about the same as before. Notice that the $35: 10: 1$ forecast is from the committee, but does not appear to smooth the data series' sharp transitions.

In Figure (c), the 35:2:1 network is not included because of its poor forecasting performance on the more noisy data series. The 35:10:1 and 35:20:1 forecasts are slightly worse than before.

In Figure (d), the 35:2:1 network is included and its coefficient of determination is much improved from before. The 35:10:1 and 35:20:1 network forecasts are decent, despite the low coefficient of determination for 35:10:1. The forecasts appear to be shifted up when compared to those in Error! Reference source not found. (d).

Finally, by evaluating the charts in Figure and data in Table some observations can be made:

The total squared error and unscaled error are higher for noisy data series with the exception of the 35:10:1 network trained on the noisier data series. It trained to extremely low errors, orders of magnitude lower than with the heuristic, but its coefficient of determination is also lower. This is probably an indication of overfitting the noisier data series with simple training, which hurt its forecasting performance.

The errors do not appear to correlate well with the coefficient of determination.

In most cases, the committee forecast is worse than the best candidate's forecast.

There are four networks whose coefficient of determination is negative, compared with two for the heuristic training method. 
(a)

(b)

(c)

(d)

Figure 3 The one-period forecasting accuracy for the best candidates for simply trained 35:2:1, 35:10:1, and 35:20:1 networks trained on the (a) original, (b) less noisy, (c) more noisy, and (d) ascending data series.

(a)

(b)

(c)

Figure 4 Graphical comparison of metrics for the networks in Figure : (a) total squared error, (b) unscaled error, and (c) coefficient of determination. Note that the vertical axis is logarithmic for (a) and (b). The raw data are given in Table .

Table 3 Parameters and metrics for the networks in Figure .

\begin{tabular}{|l|l|l|l|l|l|l|l|l|}
\hline Arch. & Candidate & $\begin{array}{l}\text { Data } \\
\text { Series }\end{array}$ & $\begin{array}{l}\text { Ending } \\
\text { Learnin } \\
\text { g } \\
\text { Rate }\end{array}$ & Epochs & $\begin{array}{l}\text { Total } \\
\text { Squared } \\
\text { Error }\end{array}$ & $\begin{array}{l}\text { Unscaled } \\
\text { Error }\end{array}$ & $\begin{array}{l}\text { Coeff. of } \\
\text { Determ. } \\
\text { (1 period) }\end{array}$ & $\begin{array}{l}\text { Training } \\
\text { Time } \\
\text { (sec. })\end{array}$ \\
\hline $35: 2: 1$ & b & Original & 0.1 & 100000 & 0.0035864 & 14.6705 & 0.9111 & 99 \\
\hline $35: 10: 1$ & c & Original & 0.1 & 100000 & $3.02316 \mathrm{e}-006$ & 0.386773 & 1.0000 & 286 \\
\hline $35: 20: 1$ & a & Original & 0.1 & 100000 & $2.15442 \mathrm{e}-006$ & 0.376312 & 1.0000 & 515 \\
\hline $35: 2: 1$ & $\mathrm{~b}$ & Less Noisy & 0.1 & 100000 & 0.0822801 & 84.8237 & 0.5201 & 99 \\
\hline $35: 10: 1$ & committee (b) & Less Noisy & 0.1 & 100000 & 0.00341762 & 17.6535 & 0.9173 & 287 \\
\hline $35: 20: 1$ & $\mathrm{~b}$ & Less Noisy & 0.1 & 100000 & 0.00128001 & 10.8401 & 0.9453 & 531 \\
\hline $35: 2: 1$ & $\mathrm{~b}$ & $\begin{array}{l}\text { More } \\
\text { Noisy }\end{array}$ & 0.1 & 100000 & 0.360209 & 203.893 & -4.6748 & 100 \\
\hline $35: 10: 1$ & $\mathrm{a}$ & $\begin{array}{l}\text { More } \\
\text { Noisy }\end{array}$ & 0.1 & 100000 & $2.47609 \mathrm{e}-009$ & 0.0166514 & -0.0056 & 282 \\
\hline $35: 20: 1$ & $\mathrm{c}$ & $\begin{array}{l}\text { More } \\
\text { Noisy }\end{array}$ & 0.1 & 100000 & 0.000106478 & 3.65673 & -1.5032 & 519 \\
\hline $35: 2: 1$ & $\mathrm{a}$ & Ascending & 0.1 & 100000 & 0.023301 & 59.7174 & 0.4091 & 98 \\
\hline $35: 10: 1$ & $\mathrm{a}$ & Ascending & 0.1 & 100000 & 0.000421792 & 8.67945 & -0.3585 & 280 \\
\hline $35: 20: 1$ & $\mathrm{~b}$ & Ascending & 0.1 & 100000 & 0.000191954 & 5.87395 & 0.4154 & 529 \\
\hline
\end{tabular}


and Error! Reference source not found. list beginning parameters for all neural networks trained with the heuristic algorithm and simple method, respectively. Parameters for trained networks (e.g., the actual number of training epochs) are presented in Section Error! Reference source not found. and Section Error! Reference source not found.

Often, two trained networks with identical beginning parameters can produce drastically different forecasts. This is because each network is initialized with random weights and biases prior to training, and during training, networks converge to different minima on the training error curve. Therefore, three candidates of each network configuration were trained. This allows another neural network forecasting technique to be used: forecasting by committee. In forecasting by committee, the forecasts from the three networks are averaged together to produce a new forecast. This may either smooth out noisy forecasts or introduce error from a poorly performing network. The coefficient of determination will be calculated for the three networks' forecasts and for the committee forecast to determine the best candidate.

Table 1Beginning parameters for heuristically trained neural networks.

\begin{tabular}{|c|c|c|c|c|c|c|}
\hline \multicolumn{7}{|c|}{$\begin{array}{l}\text { Heuristic Algorithm Training } \\
\text { Update Frequency }=50 \text {, Change Frequency }=10, \text { Decrement }=0.05\end{array}$} \\
\hline Architecture & $\begin{array}{l}\text { Learning } \\
\text { Rate }\end{array}$ & $\begin{array}{l}\text { Epochs } \\
\text { Limit }\end{array}$ & $\begin{array}{l}\text { Error } \\
\text { Limit }\end{array}$ & $\begin{array}{l}\text { Data Series } \\
O=\text { original } \\
\mathbf{L}=\text { less noisy } \\
\mathbf{M}=\text { more noisy } \\
\mathrm{A}=\text { ascending }\end{array}$ & $\begin{array}{l}\text { Training } \\
\text { Data Set } \\
\text { Range (\# } \quad \text { Point } \\
\text { Examples) }\end{array}$ & $\begin{array}{l}\text { Validation Set } \\
\text { Data Point Range } \\
\text { (\# of Examples) }\end{array}$ \\
\hline $35: 20: 1$ & 0.3 & 500,000 & $1 \times 10^{-10}$ & $\mathrm{O}, \mathrm{L}, \mathrm{M}, \mathrm{A}$ & $0-143(109)$ & $144-215(37)$ \\
\hline $35: 10: 1$ & 0.3 & 500,000 & $1 \times 10^{-10}$ & $\mathrm{O}, \mathrm{L}, \mathrm{M}, \mathrm{A}$ & $0-143(109)$ & $144-215(37)$ \\
\hline $35: 2: 1$ & 0.3 & 500,000 & $1 \times 10^{-10}$ & $\mathrm{O}, \mathrm{L}, \mathrm{M}, \mathrm{A}$ & $0-143(109)$ & $144-215(37)$ \\
\hline $25: 20: 1$ & 0.3 & 250,000 & $1 \times 10^{-10}$ & $\mathrm{O}$ & $0-143(119)$ & $144-215(47)$ \\
\hline $25: 10: 1$ & 0.3 & 250,000 & $1 \times 10^{-10}$ & $\mathrm{O}$ & $0-143(119)$ & $144-215(47)$ \\
\hline
\end{tabular}

Table 2 beginning parameter

\begin{tabular}{|l|l|l|l|l|l|l|}
\hline Simple Method Training & Learning & $\begin{array}{l}\text { Epochs } \\
\text { Limit }\end{array}$ & $\begin{array}{l}\text { Error } \\
\text { Limit }\end{array}$ & $\begin{array}{l}\text { Data Series } \\
\text { O= original } \\
\text { L= less noisy } \\
\text { M= more noisy } \\
\text { A= ascending } \\
\text { S= sunspots }\end{array}$ & $\begin{array}{l}\text { Training } \\
\text { Data } \\
\text { Range } \\
\text { Examples) }\end{array}$ & $\begin{array}{c}\text { Set } \begin{array}{l}\text { Point } \\
\text { of }\end{array} \\
\text { Validation } \\
\text { Data Point } \\
\text { (\# of Examples) }\end{array}$ \\
\hline $35: 20: 1$ & 0.1 & 100,000 & $1 \times 10^{-10}$ & O, L, M, A & $0-143(109)$ & $144-215(37)$ \\
\hline $35: 10: 1$ & 0.1 & 100,000 & $1 \times 10^{-10}$ & O, L, M, A & $0-143(109)$ & $144-215(37)$ \\
\hline $35: 2: 1$ & 0.1 & 100,000 & $1 \times 10^{-10}$ & O, L, M, A & $0-143(109)$ & $144-215(37)$ \\
\hline $30: 30: 1$ & 0.05 & 100,000 & $1 \times 10^{-10}$ & S & $0-165(136)$ & - \\
\hline
\end{tabular}

Often, two trained networks with identical beginning parameters can produce drastically different forecasts.
This is because each network is initialized with random weights and biases prior to training, and during training, networks converge to different minima on the training error curve. Therefore, three 
candidates of each network configuration were trained. This allows another neural network forecasting technique to be used: forecasting by committee. In forecasting by committee, the forecasts from the three networks are averaged together to produce a new forecast. This may either smooth out noisy forecasts or introduce error from a poorly performing network. The coefficient of determination will be calculated for the three networks' forecasts and for the committee forecast to determine the best candidate.

\section{Multi Neural Network Parameters and Procedure Architectures}

To test how feed-forward neural networks respond to various data series, a network architecture that could accurately learn and model the original series is needed. An understanding of the feed-forward neural network is necessary to specify the number of network inputs. The trained network acts as a function: given a set of inputs it calculates an output. The network does not have any concept of temporal position and cannot distinguish between identical sets of inputs with different actual outputs. For example, referencing the original data series, if there are ten network inputs, among the training examples (assuming the training set covers an entire period) there are several instances where two examples have equivalent input vectors but different output vectors. One such instance is shown in Figure . This may "confuse" the network during training, and fed that input vector during forecasting, the network's output may be somewhere in-between the output vectors' values or simply "way off”!

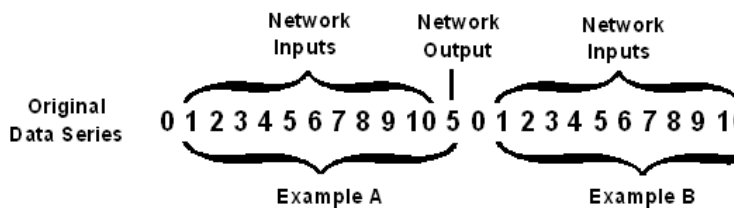

Figure 2 One instance in the original data series where two examples have equivalent input vectors but different output vectors.

Inspection of the original data series reveals that a network with at least twenty-four inputs is required to make unambiguous examples. (Given twenty-three inputs, the largest ambiguous example input vectors would be from zero-based data point 10 to 32 and from 34 to 56.) Curiously, considerably more network inputs are required to make good forecasts, as will be seen in Section Error! Reference source not found.

Next, through trial-and-error the number of hidden layers was found to be one and the number of units in that layer was found to be ten for the artificial data series. The number of output layer units is necessarily one. This network, called 35:10:1 for shorthand, showed excellent forecasting performance on the original data series, and was selected to be the reference for comparison and evaluation. To highlight any effects of having a too-small or too-large network for the task, two other networks, 35:2:1 and 35:20:1, respectively, are also included in the evaluation. Also, addressing the curiosity raised in the previous paragraph, two more networks, 25:10:1 and 25:20:1, are included.

It is much more difficult to choose the appropriate number of network inputs for the sunspots data series. By inspecting the data series and through trial-anderror, thirty network inputs were selected. Also through trial-and-error, one hidden layer with thirty units was selected. Although this network seems excessively large, it proved to be the best neural network forecaster for the sunspots data series. Other networks that were tried include 10:10:1, 20:10:1, 30:10:1, 10:20:1, 20:20:1, and 30:20:1.

\section{Training}

By observing neural network training characteristics, a heuristic algorithm was developed and implemented in FORECASTER. The parameters for the heuristic are set within the Training Parameters dialog (see Section Error! Reference source not found.). The heuristic requires the user to set the learning rate and epochs limit to higher-than-normal values (e.g., 0.3 and 500,000 , respectively) and the error limit to a lowerthan-normal value (e.g., $1 \times 10^{-10}$ ). The heuristic also uses three additional user-set parameters: the number of training epochs before an application window (view) update (update frequency), the number of updates before a learning rate change (change frequency), and a learning rate change decrement (decrement). Finally, the heuristic requires the data series to be partitioned into a training set and validation set. Given these users set parameters, the heuristic algorithm is: 
for each view-update during training

if the validation error is higher than the lowest value seen

increment count
if count equals change-frequency
if the learning rate minus decrement is
greater than zero
lower the learning rate by
decrement
reset count
continue
else
stop training

The purpose of the heuristic is to start with an aggressive learning rate, which will quickly find a coarse solution, and then to gradually decrease the learning rate to find a finer solution. Of course, this could be done manually by observing the validation error and using the Change Training Parameters dialog to alter the learning rate. But an automated solution is preferred, especially for an empirical evaluation.

In the evaluation, the heuristic algorithm is compared to the "simple" method of training where training continues until either the number of epochs grows to the epochs limit or the total squared error drops to the error limit. Networks trained with the heuristic algorithm are termed "heuristically trained"; networks trained with the simple method are termed "simply trained".

Finally, the data series in Section Error! Reference source not found. are partitioned so that the training set is the first two periods and the validation set is the third period. Note that "period" is used loosely for less noisy, noisier, and ascending, since they are not strictly periodic.

\section{Simply Trained Neural Networks with Thirty-Five Inputs}

Figure graphically shows the one-period forecasting accuracy for the best candidates for simply trained 35:2:1, 35:10:1, and 35:20:1 networks. Figure (a), (b), and (c) show forecasts from networks trained on the original, less noisy, and more noisy data series, respectively, and the forecasts are compared to a period of the original data series. Figure (d) shows forecasts from networks trained on the ascending data series, and the forecasts are compared to a fourth "period" of the ascending series. In Figure (c) the 35:2:1 network is not included.

Figure graphically compares metrics for the best candidates for simply trained 35:2:1, 35:10:1, and 35:20:1 networks. Figure (a) and (b) compare the total squared error and unscaled error, respectively, and (c) compares the coefficient of determination. The vertical axis in (a) and (b) is logarithmic. The number of epochs and training time are not included in Figure because all networks were trained to 100,000 epochs.

Finally, Table lists the raw data for the charts in Figure and other parameters used in training. Refer to Error! Reference source not found. for more parameters.

In this case, it seems the heuristic was inappropriate. Notice that the heuristic allowed the 35:2:1 network to train for 28,850 epochs more than the simple method. Also, the total squared error and unscaled error for the heuristically trained network were lower, but so was the coefficient of determination it was much lower. Again, the forecasts for the 35:10:1 and 35:20:1 networks are near perfect, and are indistinguishable from the original data series.

In Figure (b) the 35:2:1 network forecast is worse than in Error! Reference source not found. (b), whereas the 35:10:1 and 35:20:1 forecasts are about the same as before. Notice that the $35: 10: 1$ forecast is from the committee, but does not appear to smooth the data series' sharp transitions.

In Figure (c), the 35:2:1 network is not included because of its poor forecasting performance on the more noisy data series. The 35:10:1 and 35:20:1 forecasts are slightly worse than before.

In Figure (d), the 35:2:1 network is included and its coefficient of determination is much improved from before. The 35:10:1 and 35:20:1 network forecasts are decent, despite the low coefficient of determination for $35: 10: 1$. The forecasts appear to be shifted up when compared to those in Error! Reference source not found. (d). 
Finally, by evaluating the charts in Figure and data in Table some observations can be made:

The total squared error and unscaled error are higher for noisy data series with the exception of the 35:10:1 network trained on the noisier data series. It trained to extremely low errors, orders of magnitude lower than with the heuristic, but its coefficient of determination is also lower. This is probably an indication of overfitting the noisier data series with simple training, which hurt its forecasting performance.

The errors do not appear to correlate well with the coefficient of determination.

In most cases, the committee forecast is worse than the best candidate's forecast.

There are four networks whose coefficient of determination is negative, compared with two for the heuristic training method. 


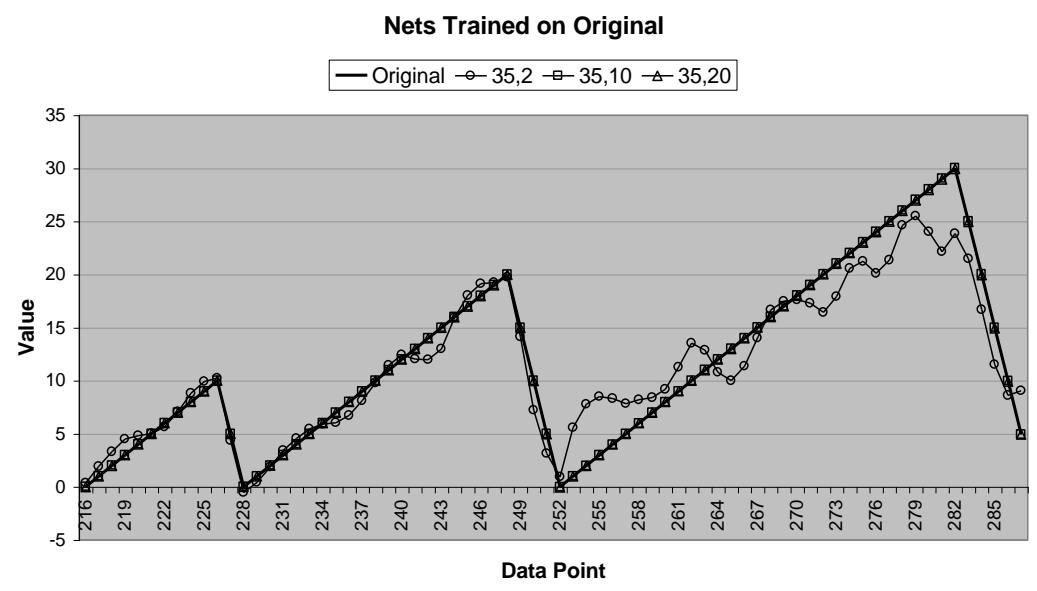

(a)

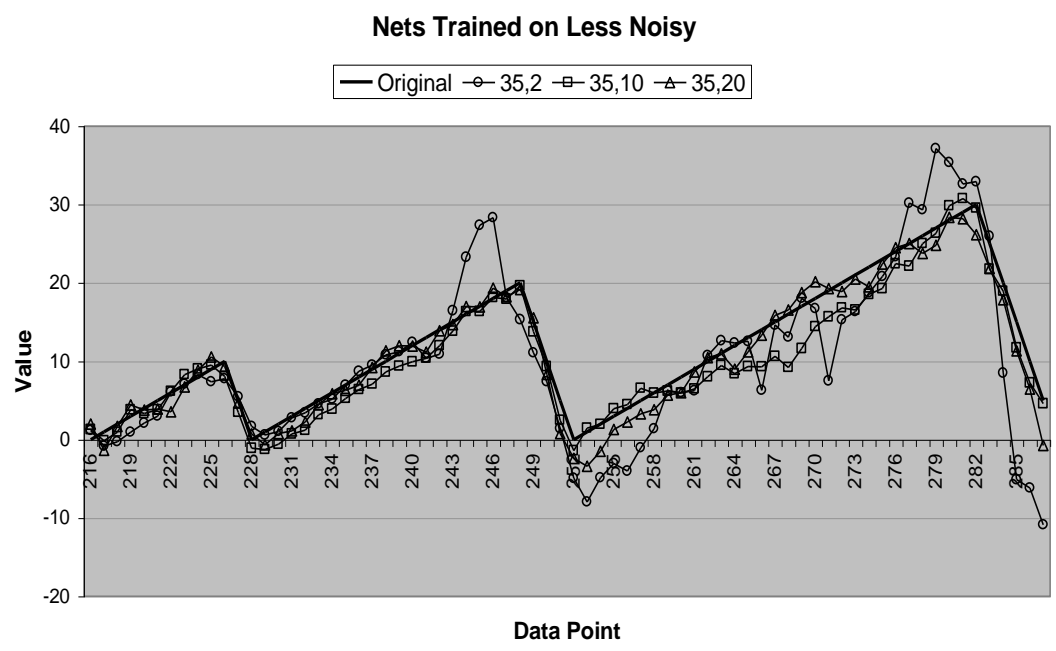

(b)

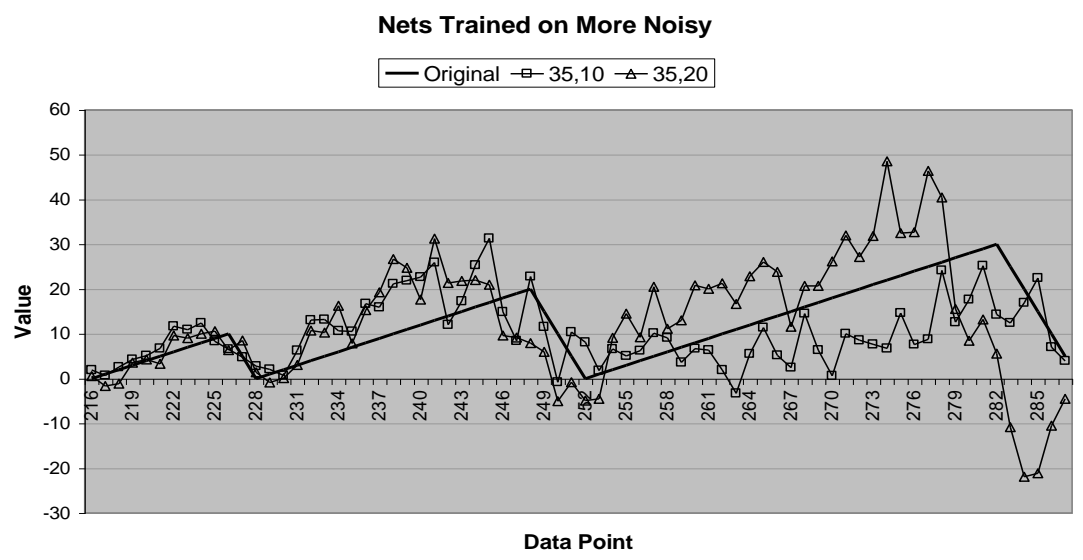

(c) 


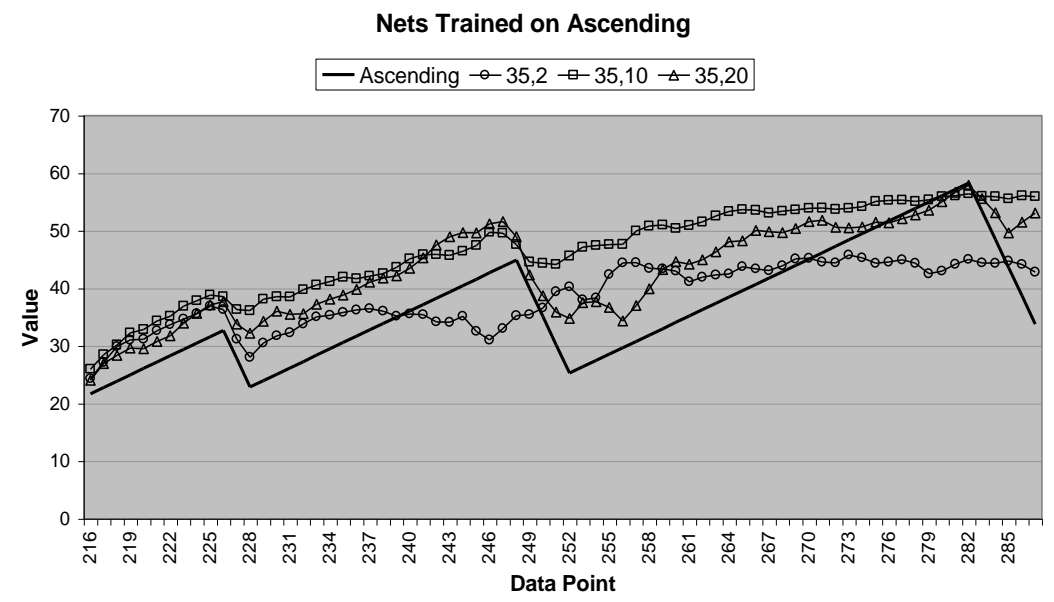

(d)

Figure 3 The one-period forecasting accuracy for the best candidates for simply trained 35:2:1, 35:10:1, and 35:20:1 networks trained on the (a) original, (b) less noisy, (c) more noisy, and (d) ascending data series.

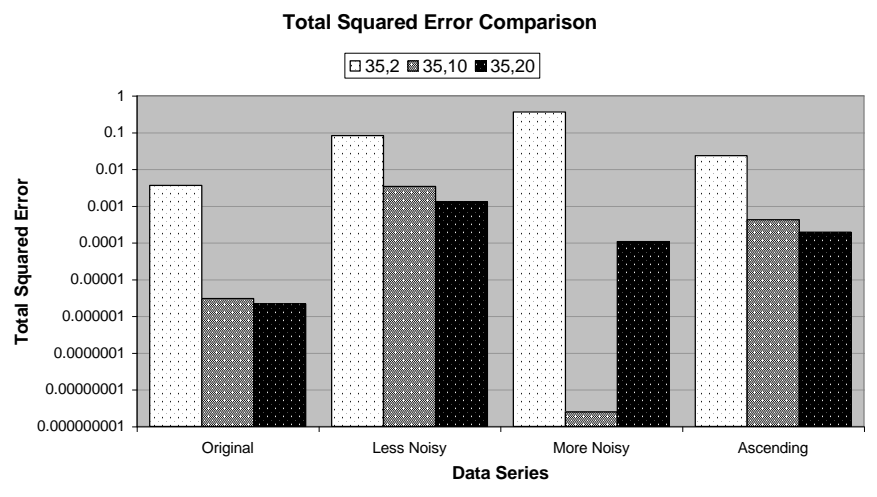

(a)

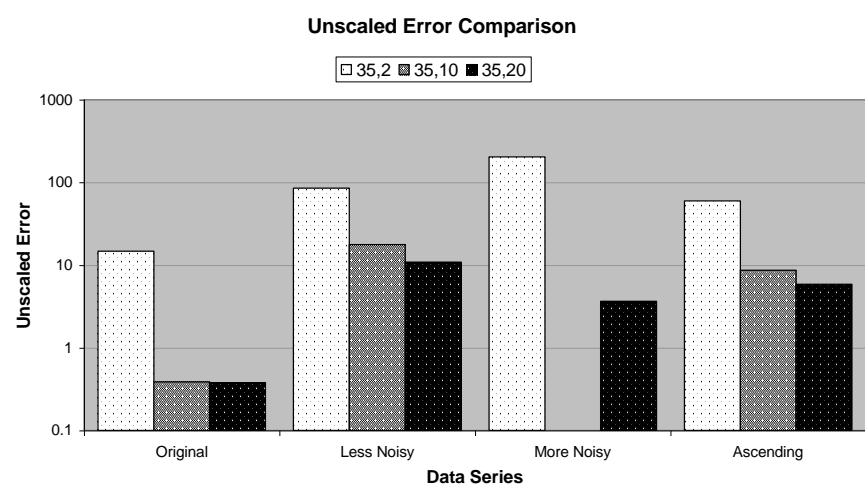

(b)

Coefficient of Determination Comparison

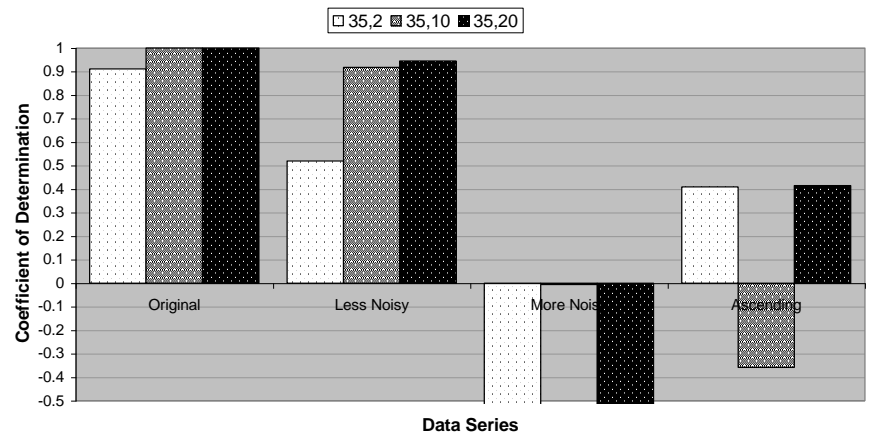

(c)

Figure 4 Graphical comparison of metrics for the networks in Figure : (a) total squared error, (b) unscaled error, and (c) coefficient of determination. Note that the vertical axis is logarithmic for (a) and (b). The raw data are given in Table . 
Table 3 Parameters and metrics for the networks in Figure .

\begin{tabular}{|l|l|l|l|l|l|l|l|l|}
\hline Arch. & Candidate & $\begin{array}{l}\text { Data } \\
\text { Series }\end{array}$ & $\begin{array}{l}\text { Ending } \\
\text { Learnin } \\
\text { g } \\
\text { Rate }\end{array}$ & Epochs & $\begin{array}{l}\text { Total } \\
\text { Squared } \\
\text { Error }\end{array}$ & $\begin{array}{l}\text { Unscaled } \\
\text { Error }\end{array}$ & $\begin{array}{l}\text { Coeff. of } \\
\text { Determ. } \\
\text { (1 period) }\end{array}$ & $\begin{array}{l}\text { Training } \\
\text { Time } \\
\text { (sec. })\end{array}$ \\
\hline $35: 2: 1$ & b & Original & 0.1 & 100000 & 0.0035864 & 14.6705 & 0.9111 & 99 \\
\hline $35: 10: 1$ & $\mathrm{c}$ & Original & 0.1 & 100000 & $3.02316 \mathrm{e}-006$ & 0.386773 & 1.0000 & 286 \\
\hline $35: 20: 1$ & $\mathrm{a}$ & Original & 0.1 & 100000 & $2.15442 \mathrm{e}-006$ & 0.376312 & 1.0000 & 515 \\
\hline $35: 2: 1$ & $\mathrm{~b}$ & Less Noisy & 0.1 & 100000 & 0.0822801 & 84.8237 & 0.5201 & 99 \\
\hline $35: 10: 1$ & committee (b) & Less Noisy & 0.1 & 100000 & 0.00341762 & 17.6535 & 0.9173 & 287 \\
\hline $35: 20: 1$ & $\mathrm{~b}$ & Less Noisy & 0.1 & 100000 & 0.00128001 & 10.8401 & 0.9453 & 531 \\
\hline $35: 2: 1$ & $\mathrm{~b}$ & $\begin{array}{l}\text { More } \\
\text { Noisy }\end{array}$ & 0.1 & 100000 & 0.360209 & 203.893 & -4.6748 & 100 \\
\hline $35: 10: 1$ & $\mathrm{a}$ & $\begin{array}{l}\text { More } \\
\text { Noisy }\end{array}$ & 0.1 & 100000 & $2.47609 \mathrm{e}-009$ & 0.0166514 & -0.0056 & 282 \\
\hline $35: 20: 1$ & $\mathrm{c}$ & $\begin{array}{l}\text { More } \\
\text { Noisy }\end{array}$ & 0.1 & 100000 & 0.000106478 & 3.65673 & -1.5032 & 519 \\
\hline $35: 2: 1$ & $\mathrm{a}$ & Ascending & 0.1 & 100000 & 0.023301 & 59.7174 & 0.4091 & 98 \\
\hline $35: 10: 1$ & $\mathrm{a}$ & Ascending & 0.1 & 100000 & 0.000421792 & 8.67945 & -0.3585 & 280 \\
\hline $35: 20: 1$ & $\mathrm{~b}$ & Ascending & 0.1 & 100000 & 0.000191954 & 5.87395 & 0.4154 & 529 \\
\hline
\end{tabular}

\section{REFERENCES}

[1] M. Abdollahzade, A. Miranian, H. Hassani, and H. Iranmanesh, "A new hybrid enhanced local linear neuro-fuzzy model based on the optimized singular spectrum analysis and its application for nonlinear and chaotic time series forecasting," Inf. Sci. (Ny)., vol. 295, pp. 107-125, 2015.

[2] J. Ares, J. A. Lara, D. Lizcano, and S. Suárez, "A soft computing framework for classifying time series based on fuzzy sets of events," Inf. Sci. (Ny)., vol. 330, pp. 125-144, 2016.

[3] M.-Y. Chen and B.-T. Chen, "A hybrid fuzzy time series model based on granular computing for stock price forecasting," Inf. Sci. (Ny)., vol. 294, pp. 227$241,2015$.

[4] T.-T. Chen and S.-J. Lee, "A weighted LS-SVM based learning system for time series forecasting," Inf. Sci. (Ny)., vol. 299, pp. 99-116, 2015.

[5] Z. Chen, W. Zuo, Q. Hu, and L. Lin, "Kernel sparse representation for time series classification," Inf. Sci. (Ny)., vol. 292, pp. 15-26, 2015.

[6] S.-H. Cheng, S.-M. Chen, and W.-S. Jian, "Fuzzy time series forecasting based on fuzzy logical relationships and similarity measures," Inf. Sci. (Ny)., vol. 327, pp. 272-287, 2016.

[7] H. Deng, G. Runger, E. Tuv, and M. Vladimir, "A time series forest for classification and feature extraction,” Inf. Sci. (Ny)., vol. 239, pp. 142-153, 2013.
[8] L. N. Ferreira and L. Zhao, "Time series clustering via community detection in networks," Inf. Sci. (Ny)., vol. 326, pp. 227-242, 2016.

[9] M. González, C. Bergmeir, I. Triguero, Y. Rodríguez, and J. M. Benítez, "On the stopping criteria for $\mathrm{k}$ Nearest Neighbor in positive unlabeled time series classification problems," Inf. Sci. (Ny)., vol. 328, pp. 42-59, 2016.

[10] X. Huang, Y. Ye, L. Xiong, R. Y. K. Lau, N. Jiang, and S. Wang, "Time series k-means: A new k-means type smooth subspace clustering for time series data,” Inf. Sci. (Ny)., vol. 367-368, pp. 1-13, 2016.

[11] A. Kattan, S. Fatima, and M. Arif, "Time-series event-based prediction: An unsupervised learning framework based on genetic programming," Inf. Sci. (Ny)., vol. 301, pp. 99-123, 2015.

[12] M. Krawczak and G. Szkatuła, "An approach to dimensionality reduction in time series," Inf. Sci. (Ny)., vol. 260, pp. 15-36, 2014.

[13] L. Liu, Y. Peng, S. Wang, M. Liu, and Z. Huang, "Complex activity recognition using time series pattern dictionary learned from ubiquitous sensors," Inf. Sci. (Ny)., vol. 340, pp. 41-57, 2016.

[14] A. Marszałek and T. Burczyński, "Modeling and forecasting financial time series with ordered fuzzy candlesticks," Inf. Sci. (Ny)., vol. 273, pp. 144-155, 2014.

[15] S. Miao, U. Vespier, R. Cachucho, M. Meeng, and A. Knobbe, "Predefined pattern detection in large time series," Inf. Sci. (Ny)., vol. 329, pp. 950-964, 2016. 
[16] V. Novák, I. Perfilieva, M. Holčapek, and V. Kreinovich, "Filtering out high frequencies in time series using F-transform," Inf. Sci. (Ny)., vol. 274, pp. 192-209, 2014.

[17] H. Pree, B. Herwig, T. Gruber, B. Sick, K. David, and P. Lukowicz, "On general purpose time series similarity measures and their use as kernel functions in support vector machines," Inf. Sci. (Ny)., vol. 281, pp. 478-495, 2014.

[18] M. Pulido, P. Melin, and O. Castillo, "Particle swarm optimization of ensemble neural networks with fuzzy aggregation for time series prediction of the Mexican Stock Exchange," Inf. Sci. (Ny)., vol. 280, pp. 188204, 2014.

[19] T. Xiong, Y. Bao, Z. Hu, and R. Chiong, "Forecasting interval time series using a fully complex-valued RBF neural network with DPSO and PSO algorithms," Inf. Sci. (Ny)., vol. 305, pp. 77-92, 2015.

[20] F. Ye, L. Zhang, D. Zhang, H. Fujita, and Z. Gong, "A novel forecasting method based on multi-order fuzzy time series and technical analysis," Inf. Sci. (Ny)., vol. 367-368, pp. 41-57, 2016.

[21] H. Zhao, Z. Dong, T. Li, X. Wang, and C. Pang, "Segmenting time series with connected lines under maximum error bound," Inf. Sci. (Ny)., vol. 345, pp. $1-8,2016$.

[22] S. Zhu, Q.-L. Han, and C. Zhang, "Investigating the effects of time-delays on stochastic stability and designing 11-gain controllers for positive discretetime Markov jump linear systems with time-delay," Inf. Sci. (Ny)., vol. 355, pp. 265-281, 2016. 